\title{
Pertinence des critères d'évaluation utilisés dans les essais cliniques sur la maladie d'Alzheimer
}

\author{
Philippe Truffinet ${ }^{1}$, Régis Bordet ${ }^{2}$, Joël Ménard ${ }^{3}$ et les participants à la table ronde $n^{\circ} 1$ de Giens XXIV \\ 1 Sanofi Aventis, Antony, France \\ 2 EA 1046, Faculté de Médecine, Université de Lille II, Département Universitaire de Pharmacologie, Lille, France \\ 3 SPIM, Faculté de Médecine René Descartes, Université Paris V, Paris, France
}

Texte reçu le 15 octobre 2008 ; accepté le 20 novembre 2008

\section{Mots clés : \\ Alzheimer; \\ évaluation; \\ étude clinique; \\ biomarqueur}

Résumé - Le travail de la table ronde (TR) $n^{\circ} 1$ «Pertinence des critères d'évaluation dans les essais cliniques sur la maladie d'Alzheimer » a utilisé comme fil conducteur le guideline publié en juillet 2008 par l'EMEA (European Medicines Agency) pour le développement des médicaments dans la maladie d'Alzheimer et autres démences, et a porté principalement sur deux des trois indications thérapeutiques recensées dans le guideline - les traitements symptomatiques et les médicaments modifiant le cours évolutif de la maladie - à l'exclusion de l'approche de prévention primaire. La discussion s'est focalisée sur deux aspects principaux : l'amélioration de la sélection des patients dans les essais cliniques et les critères de jugements cliniques et les biomarqueurs. Les suggestions suivantes ont été formulées :

- Renforcer l'intérêt pour les études cliniques dans les phases précoces de la maladie d'Alzheimer (y compris prodromales), notamment pour les candidats médicaments modifiant le cours évolutif de la maladie.

- Renforcer l'expertise des centres de recherche avec les biomarqueurs, de façon à faciliter leur utilisation ultérieure dans les études cliniques, soit pour compléter la description des patients inclus, soit à titre de critères de sélection. De plus, les travaux en cours en France de standardisation inter-centre de neuro-imagerie et des dosages de substrats du liquide céphalo-rachidien sont essentiels pour préparer leur utilisation multicentrique.

- Favoriser la réalisation d'études ancillaires de biomarqueurs, greffées sur des études cliniques.

- Renforcer la formation et l'expérience des cotateurs avec les échelles fonctionnelles, qui doivent maintenant constituer une des deux évaluations principales des études pivotales, et avec les items additionnels de l'ADAS-cog (Alzheimer's Disease Assessment Scale, Cognitive sub scale) qui sont utiles pour les formes précoces de maladie d'Alzheimer.

- Améliorer la connaissance des propriétés des échelles cliniques fonctionnelles, par l'analyse détaillée des bases de données disponibles d'études cliniques, dans des collaborations public/privé.

- Améliorer la connaissance des correspondances entre échelles utilisées dans les études cliniques et outils utilisés dans la pratique quotidienne (généralement différents), de façon à étayer l'interprétation de la pertinence clinique des résultats d'études cliniques.

- Réfléchir aux éventuels besoins d'adaptation des outils d'évaluation clinique aux évolutions sociétales, notamment pour les évaluations des performances cognitives.

- Discuter la possibilité de mesures de protections supplémentaires des données pour les médicaments candidats antiAlzheimer médicaments modifiant le cours évolutif de la maladie étant donné l'impact négatif pour tous les acteurs des contraintes de durée et difficultés des essais thérapeutiques.

- Rediscuter l'éthique et l'acceptabilité potentielle d'études contre placebo en monothérapie sur des durées de 6-9 mois.

\footnotetext{
* Pour la liste des participants, voir en fin d'article.
} 


\section{Introduction}

La table ronde $(\mathrm{TR}) \mathrm{n}^{\circ} 1$ « Pertinence des critères d'évaluation dans les essais cliniques sur la maladie d'Alzheimer» s'est inscrite dans un contexte international de développement de molécules innovantes par les firmes pharmaceutiques et de recherche de cibles thérapeutiques au niveau académique. L'ampleur du problème de santé publique que représente la maladie d'Alzheimer est bien documenté $:^{[1,2]}$ pathologie très fréquente, affectant un nombre croissant de sujets du fait du vieillissement de la population, et dont la sévérité se manifeste non seulement par ses effets sur le fonctionnement de la personne mais également par le poids qu'elle fait peser sur l'entourage... Malgré une activité intense de recherche et développement de nouvelles thérapeutiques, les médicaments disponibles restent peu nombreux : trois médicaments inhibant l'acétylcholinestérase, et un bloqueur des récepteurs glutamatergiques NMDA. De plus, les débats concernant leur ratio coût bénéfice sont actuellement vifs, avec des controverses sur les recommandations de remboursement dans certains pays. Les développements de nouveaux traitements ont à affronter de multiples difficultés, dont témoigne le nombre d'échecs récents, y compris au stade avancé d'études cliniques pivotales chez les patients.

L'opportunité de la TR a été renforcée par le plan gouvernemental sur la maladie d'Alzheimer, qui a pour objectif de fédérer la recherche dans ce domaine à l'échelon national. Le contexte est aussi celui du maintien de l'attractivité de la France pour la réalisation des essais cliniques dans un domaine où elle a eu une position historiquement forte. Le travail de la TR s'est référé à la publication en juillet 2008, par l'EMEA, du guideline européen pour le développement des médicaments dans la maladie d'Alzheimer et autres démences. ${ }^{[3]}$ L'analyse de ce nouveau guideline a servi de fil conducteur au travail de la TR, en mettant en lumière les évolutions par rapport au guideline précédent (de 1997). ${ }^{[4]}$ Les travaux de la TR ont porté principalement sur deux des trois approches thérapeutiques recensées dans le guideline les traitements symptomatiques et les médicaments modifiant le cours évolutif de la maladie - à l'exclusion de l'approche de prévention primaire. La discussion s'est focalisée sur deux aspects principaux : l'amélioration de la sélection des patients dans les études cliniques, et les critères de jugements cliniques et les biomarqueurs.

\section{Des recommandations en évolution}

Les agences réglementaires ont voulu, depuis les années 1990, cadrer les développements de nouvelles thérapies de la maladie d'Alzheimer par des recommandations. La FDA (Food and Drug Administration) tout d'abord a publié un guideline en 1990, qui est resté à l'état de «draft », ${ }^{[5]}$ et qui introduit notamment l'exigence d'un double critère principal d'évaluation : cognitif, la cible attendue étant les fonctions cognitives, et global ou fonctionnel, pour attester que l'effet a une pertinence clinique : «To gain an antidementia indication for a product, a sponsor must provide substantial evidence that the product 1) has a clinically meaningful effect and 2) exerts its effect on the "core" manifestations of dementia. This compound requirement can be met by showing, in more than one adequate and well controlled clinical investigation, that the drug product is superior to an appropriate control treatment on both 1) a global assessment performed by a skilled clinician and 2) a performance based, objective test instrument providing a comprehensive assessment of cognitive functions. » L'EMEA, dans son guideline de 1997, reprend le principe d'un double critère principal, et étend le champ des évaluations, en ajoutant la demande d'une évaluation fonctionnelle, qui peut constituer l'une des deux évaluations principales, en alternative à l'évaluation globale. Le nouveau guideline européen conserve les principes du précédent en les étoffant (il est passé de 7 à 19 pages) et en apportant quelques évolutions. Le deuxième critère principal, aux côtés du critère cognitif, doit désormais être le critère fonctionnel (l'évaluation globale devra être un critère secondaire). Les troubles comportementaux occupent une place à part, constituant généralement des critères secondaires, dont l'évaluation est recommandée depuis le premier guideline de l'EMEA, visant à compléter la caractérisation de l'effet thérapeutique.

La définition des indications se précise avec l'évolution des recommandations. Ainsi, le guideline de la FDA reste délibérément focalisé sur le traitement maintenant qualifié de symptomatique des formes légères à modérées de démence, qui correspond à l'expérience de l'époque. Le guideline européen de 2008 formalise une catégorisation traitement symptomatique / «de fond» / préventif : "Symptomatic improvement, which may consist in enhanced cognition, more autonomy and/or improvement in neuropsychiatric and behavioural dysfunction. Disease modification with slowing or arrest of symptom progression of the dementing process. For regulatory purposes a disease modifying effect will be considered when the pharmacologic treatment delays the underlying pathological or pathophysiological disease processes and when this is accompanied by an improvement of clinical signs and symptoms of the dementing condition. Primary prevention of disease by intervention in key pathogenic mechanisms at a presymptomatic stage. »Concernant les médicaments modifiant le cours évolutif de la maladie, il est maintenant précisé que pour obtenir pleinement cette indication, des résultats « convaincants » sur des biomarqueurs permettant d'objectiver un effet sur les mécanismes neurobiologiques ou physiopathologiques de la maladie d'Alzheimer doivent être obtenus.

Le champ des indications s'élargit également d'un guideline à l'autre. Ainsi, l'on passe de recommandations sur la démence 
d'Alzheimer dans les années 1990 à un guideline en 2008 qui aborde les autres démences. De plus, le guideline admet l'intérêt du diagnostic et traitement précoces de la maladie d'Alzheimer. Critique à l'égard du concept de MCI (Mild Cognitive Impairment), considéré comme trop hétérogène, il mentionne de façon positive les travaux de recherches actuels sur le diagnostic précoce de la maladie « Currently epidemiological and clinical studies are underway to establish validated criteria for definition of «pre-dementia stages », et les critères de recherche proposés en 2007 par un groupe de travail multinational ${ }^{[6]}$ sont cités en référence.

\section{Comment optimiser la sélection des patients inclus dans les essais?}

Le diagnostic de maladie d'Alzheimer au stade de la démence repose actuellement sur les critères de démence type Alzheimer du DSM-IV, et de maladie d'Alzheimer du NINCDSADRDA (National Insitute of Neurological and Communicative Disorders and Stroke - Alzheimer's Disease and Related Disorders Association), qui sont essentiellement cliniques. Dans ces critères, l'apport des examens paracliniques se limite à l'exclusion de diagnostics différentiels. Néanmoins, ces critères manquent de spécificité, les estimations de fréquence de diagnostic erroné allant de 23 à $88 \%$. ${ }^{[6]}$ Les nombreuses recherches récentes sur des biomarqueurs ${ }^{[7,8]}$ ont montré les gains de sensibilité et spécificité qui peuvent être obtenus, notamment: (i) au plan biologique, par une ponction lombaire permettant l'analyse des concentrations d'A $\beta 1-42$, d'A $\beta 1-40$, des formes tronquées d'A $\beta$, de tau total et des formes phosphorylées de tau (Phospho-tau) et (ii) au plan de l'imagerie, par une IRM (Imagerie par Résonnance Magnétique) permettant d'objectiver l'atrophie hippocampique et l'absence (ou présence) de lésions vasculaires (lésions de substance blanche, infarctus silencieux ou micro-saignements) et d'un PETscan (Positron Emission Tomography) au FDG (Fluoro-DesoxyGlucose) permettant de monter l'hypométabolisme notamment pariéto-temporal. Les recherches ont également montré l'intérêt potentiel de ligands radio-marqués des plaques amyloïdes en PETscan, mais dont l'utilisation actuelle est encore limitée. Des critères de recherche, utilisant une sélection de ces biomarqueurs comme critères supportifs, ont été proposés. ${ }^{[6]}$

L'utilisation des biomarqueurs dans les bilans d'entrée des études cliniques reste relativement limitée, aussi bien dans les études en cours, telles que décrites sur le site internet Clinicaltrial.gov (http://www. clinicaltrials .gov/) que dans les études récemment publiées ou présentées en congrès. Les perspectives de phase II et phase III sont schématiquement à distinguer. En phase III, la sélection des patients vise classiquement à assurer une représentativité de l'échantillon par rapport à l'indication cible, et les critères cherchent donc à refléter la pratique clinique. Des techniques ne faisant pas partie de la pratique clinique courante ne répondront pas à ce but et ne seront souvent pas retenues. Néanmoins, l'utilisation de biomarqueurs peut permettre de mieux documenter le diagnostic, complétant les évaluations cliniques, qui ont une part de subjectivité, par des mesures plus objectives. C'est en phase II que le recours à des biomarqueurs peut être le plus important, dans le but de sélectionner un échantillon le plus approprié possible à la mise en évidence d'un effet thérapeutique. La TR a suggéré de systématiser l'usage de biomarqueurs pour typer les patients dans les centres de recherche, afin de renforcer leur expertise, de façon à faciliter leur utilisation ultérieure dans les études cliniques, soit pour compléter la description des patients inclus, soit à titre de critères de sélection.

Le génotypage, notamment de l'apoprotéine E, est à citer à part. Il fait de plus en plus partie des bilans d'entrée dans les études cliniques, généralement non pas dans un objectif de sélection, mais pour évaluer l'impact des génotypes testés sur la réponse thérapeutique. La pratique de génotypage a été renforcée par la publication de différences apparentes d'effet selon le génotype APO E, dans des études de rosiglitazone ${ }^{[6]}$ et de bapineuzumab (étude présentée au congrès ICAD 2008).

\section{Dessin d'étude, placebo et durée des études}

Les discussions ont principalement concerné deux difficultés des dessins des études Alzheimer : le contrôle placebo, et la durée des études pour des médicaments modifiant le cours évolutif de la maladie. La tension entre les besoins d'études contre placebo suffisamment longues et leur acceptabilité est particulièrement vive concernant les traitements symptomatiques pour lesquels des études en monothérapie sont réalisées (dans le cas de médicaments modifiant le cours évolutif de la maladie, la pratique a entériné des études en «add-on »). Les effets placebo interférant potentiellement avec les résultats d'études cliniques ont été observés avec une fréquence croissante, récemment, notamment dans les études avec traitement de moins de 6 mois de durée. Mais la communauté médicale apparait partagée concernant les pertes de chance potentielles pour les patients du fait de la non-prescription sur une durée prolongée des médicaments disponibles. En France, la durée de 6 mois, recommandée pour les études pivotales par le guideline EMEA, a souvent été considérée comme inacceptable par les investigateurs et comités d'éthiques dans les conditions de ces dernières années. Par contre, des auteurs comme L. Schneider, ${ }^{[10]}$ aux États-Unis, ont défendu l'utilisation du placebo sur cette durée. La TR a suggéré que la position des investigateurs français pourrait être rediscutée au vu des données actuellement disponibles. 
La durée des études cliniques dans le cas des «diseasemodifier » était souvent d'un an dans les années 90. Cette durée est actuellement fréquemment plus longue, de 18 mois, en ligne avec les recommandations de l'EMEA. Néanmoins, il semble, dans quelques expériences récentes, que les pentes actuelles d'évolution soient peut-être plus faibles que par le passé, et que la progression clinique soit plus lente dans les premiers 6 à 9 mois d'étude, comme dans l'étude du tarenflurbil présentée au congrès ICAD 2008. Il a été noté que ces contraintes de durée, jointes au risque très élevé d'échec, pourrait freiner les recherches à venir malgré l'ampleur des besoins médicaux, et il a été suggéré que des mesures de protection supplémentaire des données pourraient être appropriées dans ce contexte.

\section{Quels critères de jugements}

Deux points principaux ont été abordés : quelle est la pertinence des critères cliniques et leurs modalités d'application, et quels sont les paramètres qui peuvent être intégrés dans un programme de biomarqueurs. Il faut noter que les critères de jugement utilisés dans les études cliniques Alzheimer ont récemment fait l'objet d'une analyse critique détaillée par un groupe de travail organisé par l'European Alzheimer Disease Consortium (EADC). ${ }^{[11]}$

Concernant les évaluations cognitives, un débat en cours a trait à la sensibilité de l'ADAS-cog, devenue l'outil standard d'évaluation, et l'intérêt éventuel d'utiliser d'autres instruments, de développement plus récents, tels que la batterie NTB. ${ }^{[12]}$ Les avantages revendiqués de cette batterie par rapport à l'ADAScog sont l'évaluation de fonctions exécutives, et une meilleure sensibilité aux formes débutantes de la maladie. Néanmoins, les membres de la TR ont estimé que l'ADAS-cog restait un instrument qui a fait la preuve de sa sensibilité à une action pharmacologique, et que les autres instruments n'avaient pas à ce jour fait la preuve de leur supériorité. En particulier, les résultats de l'étude de phase II du bapineuzumab présentés à l'ICAD 2008 n'ont pas montré de plus grande sensibilité de NTB par rapport à ADAS-cog. Ils ont exprimé leur intérêt dans les items additionnels de l'ADAS-cog, ${ }^{[13]}$ et recommandé des entraînements et formations à ces items actuellement moins bien connus. Instrument de recherche, l'ADAS-cog n'est pas un instrument utile dans la pratique clinique. Ce décalage conduit au besoin de mieux documenter et traduire la pertinence clinique d'un bénéfice observé sur l'ADAS-cog dans des essais cliniques, par exemple par des études de corrélation entre divers instruments.

Concernant la dimension fonctionnelle, ce sont essentiellement deux échelles qui sont actuellement utilisées dans les études cliniques : 1'ADCS-ADL (Alzheimer's Disease Coopera- tive Study - Activities of Daily Living) ${ }^{[14]}$ et la DAD (Disability Assessment for Demntia). ${ }^{[15]}$ Ces échelles ont jusqu'à maintenant été utilisées en tant que critères secondaires d'évaluation, et dans ce cadre n'ont pas fait l'objet du même entraînement que les évaluations cognitives et globales. Dans les conditions actuelles d'emploi, leur variabilité apparaît comme plus grande que celle des évaluations globales. Il a donc été suggéré par la TR qu'un travail de validation et formation soit mené par les centres de recherche français, à l'instar de ce qui a été fait précédemment pour l'ADAS-cog. Il a également été recommandé de mener des actions d'entraînement des cotateurs. Par ailleurs, il a été suggéré d'utiliser les bases de données existantes pour mieux analyser les propriétés des échelles. Il est à noter que la notion de répondeur, sur des critères cognitifs et globaux ou fonctionnels, n'a pas été considérée comme appropriée car elle dichotomise, sur un seuil arbitraire et discutable, des distributions continues dans le cas de la maladie d'Alzheimer.

Les biomarqueurs, pour être utilisés en tant que critères d'évaluation dans des études cliniques, doivent avoir fait la preuve d'une évolution de scores quantitatifs sur la durée prévue de l'étude. Cette démonstration a été faite pour la volumétrie globale et hippocampique déterminée en IRM et le PET FDG (Positron Emission Tomography - Fluoro Desoxy Glucose). À ce jour, seule la volumétrie IRM a été utilisée à l'échelle d'études multicentriques pivotales. L'intérêt potentiel d'autres marqueurs dépend des mécanismes d'action étudiés, par exemple des marqueurs de la cascade amyloïde pour une action sur cette cascade. Les travaux en cours en France de standardisation inter-centre de neuroimagerie et des dosages de substrats du liquide céphalo-rachidien sont essentiels pour préparer leur utilisation multicentrique. Il est à noter que les études de cohortes, telles que l'ADNI (qui fait école, avec ses dérivations European and Japanese ADNI), qui visent à fournir des données multicentriques, n'ont pas été discutées lors de la table ronde. De plus, les études cliniques peuvent constituer un support de recherches sur les biomarqueurs, sous forme d'études ancillaires.

\section{Participants}

Serge Bakchine (CHU Reims), Luc Buée (INSERM U 422, Lille), Soraya Cherchali (Sanofi Aventis R\&D), Maylis Coste (IRI Servier), Bruno Dubois (Fédération Neurologie 4, Groupe Hospitalier Pitié Salpétrière, Paris), Françoise Duveau (Laboratoire ESAI), Jean-Marie Goehrs (Merck Sharp \& Dohme Chibret), Sylvia Goni (Laboratoires lundbeck), Philippe Gustovic (Wyeth Pharmaceuticals), Catherine Lassale (LEEM Recherche), Jean-Marc Orgogozo (CHU Bordeaux), Florence Pasquier (CHU Lille), Odile Regnier (CNAMTS), Franck Semah (CHU Lille), 
Jacques Touchon (Neurologie B, CHU Montpellier), Marc Verny (Centre de Gériatrie, Groupe Hospitalier Pitié Salpétrière, Paris), Mohammed Zaïm (Laboratoire Pierre Fabre).

\section{Références}

1. Cummings JF. Alzheimer's disease. N Engl J Med 2004; 351(1): 56-67

2. Morris JC. Dementia update 2005. Alzheimer Dis Assoc Disord 2005; 19: $100-17$

3. CHMP 2008. Guideline on medicinal products for the treatment of Alzheimer's disease and other dementias. http://www.emea.europa. eu/pdfs/human/ewp/055395en.pdf

4. EMEA. Note for guidance on medicinal products in the treatment of Alzheimer's disease. 1997. http: //www . emea. europa.eu/

5. FDA. Guidelines for the clinical evaluation of antidementia drugs, first draft, November 8, 1990. http: //www. fda.gov

6. Dubois B, Feldman HH, Jacova C, et al. Research criteria for the diagnosis of Alzheimer's disease: revising the NINCDS-ADRDA criteria. Lancet Neurol 2007; 6: 734-46

7. Shaw LM, Korecka M, Clark CM, et al. Biomarkers of neurodegeneration for diagnosis and monitoring therapeutics Nature reviews. Drug Discovery 2007; 6: 295-303

8. Blennow K. Cerebrospinal fluid protein biomarkers for Alzheimer's disease. NeuroRx 2004: 1: 213-25
9. Risner ME, Saunders AM, Altman JFB, et al. Efficacy of rosiglitazone in a genetically defined population with mild-to-moderate Alzheimer's disease. Rosiglitazone in Alzheimer's disease study group. The Pharmacogenomics Journal 2006; 6, 246-54

10. Schneider L. AD2000: donepezil in Alzheimer's disease. Lancet 2004; 363: 2100-1

11. Vellas B, Andrieu S, Sampaio C, et al. Endpoints for trials in Alzheimer's disease: a European task force consensus Lancet Neurol 2008; 7: 436-50

12. Harrison J, Minassian SL, Jenkins L, et al. A neuropsychological test battery for use in Alzheimer disease clinical trials. Arch Neurol 2007; 64(9): 1323-9

13. Mohs RC, Knopman D, Petersen RC, et al. Development of cognitive instruments for use in clinical trials of antidementia drugs: additions to the Alzheimer's disease assessment scale that broaden its scope: the Alzheimer's disease cooperative study. Alzheimer Dis Assoc Disord 1997; 11(suppl 2): S13-S21

14. Galasko D, Bennett D, Sano M, et al. A inventory to assess activities of daily living for clinical trials in Alzheimer's disease. Alzheimer Dis Assoc Disord 1997; 11 (Suppl 2): 533-9

15. Gauthier S, Gelinas I, Gauthier L. Functional disability in Alzheimer's disease. Int Psychogeriatr 1997; 9 (Suppl 1): 163-5

Correspondance et offprints : Philippe Truffinet, Sanofi Aventis, Investigations Cliniques, Neurologie, 20 rue Raymond Aron, 92165 Antony Cedex, France.

E-mail : philippe.truffinet@sanofi-aventis.com 